\title{
Glomerular fine structure in mixed membranous and proliferative glomerulonephritis
}

\author{
R. F. MACADAM
}

From the University Department of Pathology, Western Infirmary, Glasgow

SYNOPSIS A case of mixed membranous and proliferative glomerulonephritis has been studied by light and electron microscopy. Electron microscopy has shown that the cellular proliferation is mesangial in origin and that there are qualitative and quantitative alterations in the filtration basement membrane. In addition there are numerous examples of direct contact between filtration basement membrane and neutrophil polymorphonuclear leucocytes. This latter phenomenon is good direct evidence that interaction of glomerular-bound antigenantibody complex and complement has taken place. The light and electron microscopic evidence is thus in favour of the histopathological entity, mixed membranous and proliferative glomerulonephritis, being fundamentally a proliferative glomerular lesion initiated by complement-induced basement membrane injury.

\section{Materials and Methods}

The renal biopsy was obtained from a 55-year-old man with a 12-year history of nephrotic syndrome of undiagnosed aetiology. On the present admission there was mild nephrotic syndrome (blood pressure 160/100 mm $\mathrm{Hg}$ and blood urea $47 \mathrm{mg} \%$ ). The bulk of the $2.5 \mathrm{~cm}$-long pale core was fixed sequentially in $10 \%$ formol-saline and corrosive formalin and embedded in paraffin wax. Sections, $5 \mu$ thick, were stained by a variety of techniques. Cubic millimetre cortical blocks were fixed in $2 \%$ phosphate-buffered $(p \mathrm{H} \mathrm{7.4)}$ glutaraldehyde, postosmicated, and embedded in Araldite. Thin sections were doubly stained with uranyl acetate and lead citrate.

\section{Results}

LIGHT MICROSCOPY

Figure 1 is representative of all of the biopsy glomeruli. The glomerular tuft is enlarged by a considerable increase above normal in the number Received for publication 25 March 1970. of cells and a substantial intercellular accumulation of basement-membrane-like material. The inherent glomerular tuft lobularity is accentuated but patent capillaries are not restricted to, or are even common on, the tuft periphery. Figure 2 emphasizes the marked thickening of the filtering wall of the capillaries. The excess of intercapillary material appears to be definitely nonhomogeneous. There is generalized tubular epithelial cell atrophy, an increase of interstitial connective tissue, and a moderate thickening of the arterial wall.

\section{ELECTRON MICROSCOPY}

Figure 3 illustrates an intercapillary region. This is greatly increased in bulk due to an excess of intercellular material (the mesangial matrix) and an enormous population of mesangial cell cytoplasmic profiles. Some of these are extending into the adjacent parts of the filtering basement membrane. Figure 4 illustrates several features cojnmon to all glomeruli. The volume of urinary space is reduced by the increase in the glomerular tuft bulk. The epithelial cells have lost their discrete pedicles and the cytoplasm adjacent to the 
basenent membrane is denser than normal. The filtering basement membrane shows qualitative and quantitative changes. These are irregularity of thickness, an especially uneven endothelial border, a density which is generally above normal, and the presence of substantial mesangial cell cytoplasmic extensions. Mesangial cell hyperplasia causes compression of the capillary lumen and mesangial cells come into direct contact with the plasma (Fig. 5). Figure 6 shows a capillary containing two neutrophil polymorphonuclear leucocytes and a probable invading mesangial cell. These cells are in direct contact with the filtering basement membrane in the absence of an endothelial lining. This feature is illustrated at higher magnification in a different capillary (Fig. 7). The great variation in the basement membrane thickness is also easily seen in this micrograph. Maximum and minimum dimensions were $4 \mu$ and $0 \cdot 1 \mu$ respectively. Figure 8 shows the extent of the increased thickness of the basement membrane in some loops and also confirms that the mesangial cell cytoplasmic extension into the filtering basement membrane is a circumferential phenomenon. Only one example of a sub-

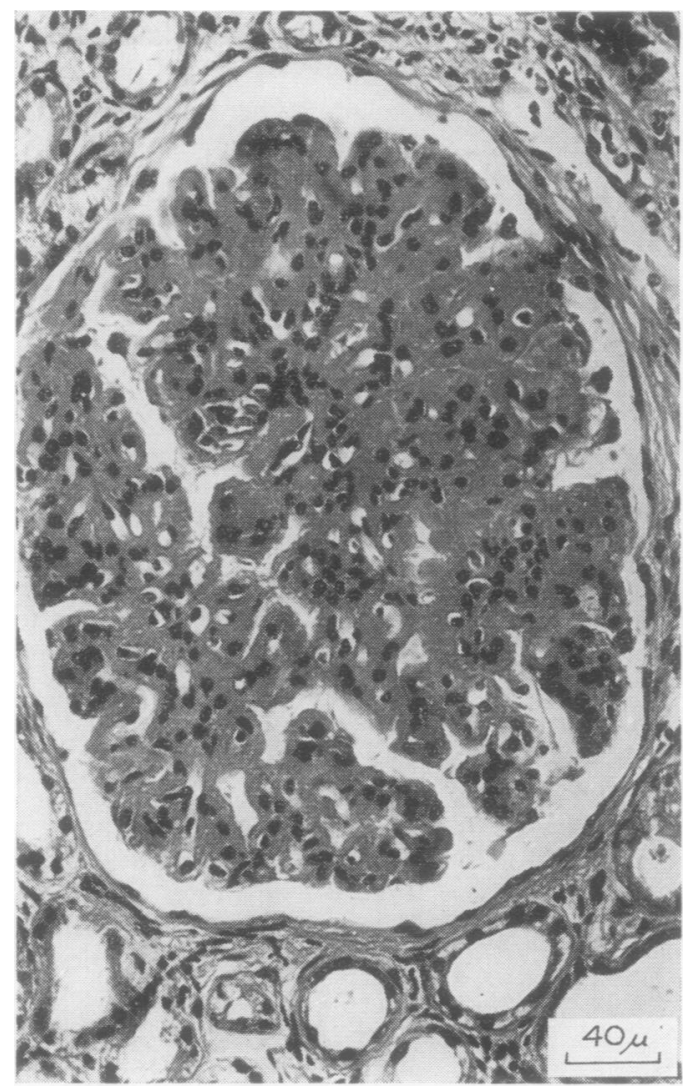

Fig. 1 Representative glomerulus of the biopsy. There is marked hypercellularity and an excess of intercellular material. Lobulation is accentuated. Haematoxylin and eosin $(H E) \times 200$. endothelial deposit was found in the entire biopsy $\frac{\varrho}{\bar{F}}$ (Fig. 9) and consisted of a very dense non- 7 homogeneous nodule.

\section{Discussion}

The glomerular histopathology in some cases of $\frac{\bar{s}}{\frac{1}{7}}$ glomerulonephritis appears like an admixture of the classical features of acute proliferative, lobular, and advanced-stage idiopathic mem- branous glomerulonephritides, and the case now:described falls into this group. Such cases have $\vec{\omega}$ been variously called (1) mixed glomerulonephritis, (2) complex glomerulonephritis, (3) un classified glomerulonephritis, and (4) mixedi్ membranous and proliferative glomeruloneph $-\vec{v}$ ritis. Kark, Pirani, Pollak, Muehrcke, andos Blainey (1958) found such a picture in 12 of $98 \mathrm{\omega}$ renal biopsies, and in a later publication (Law-으 rence, Pollak, Pirani, and Kark, 1963) inferredthat they regarded some as cases of develop-O ing lobular glomerulonephritis. Habib, Michiel-o sen, de Montera, Hinglais, Galle, and Hamburgero

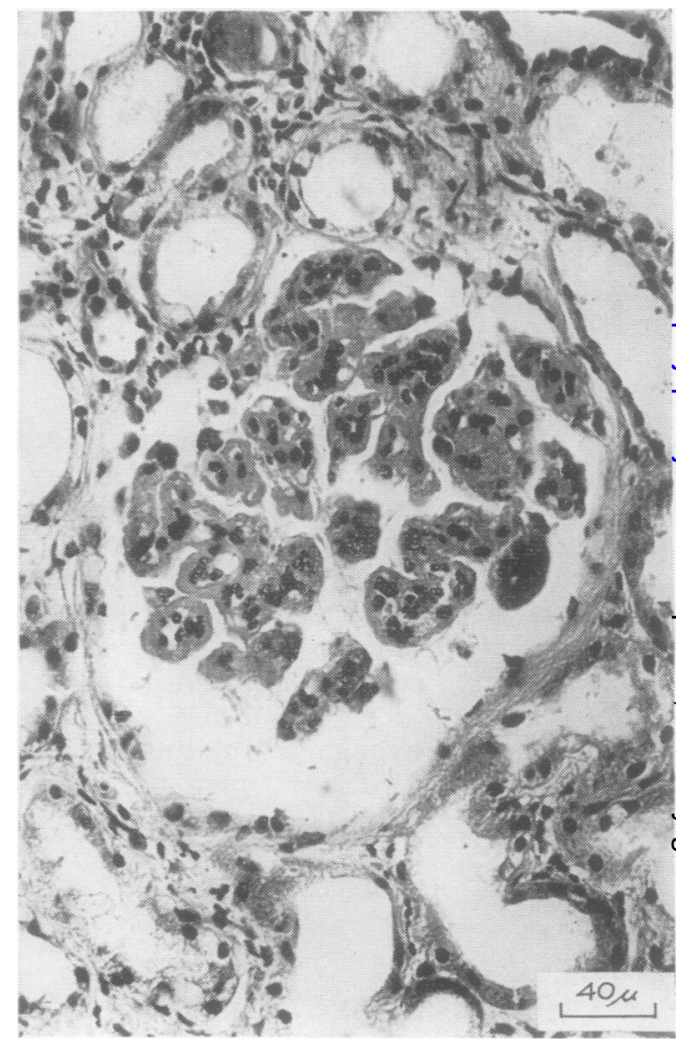

$\stackrel{\vec{\vartheta}}{0}$ 


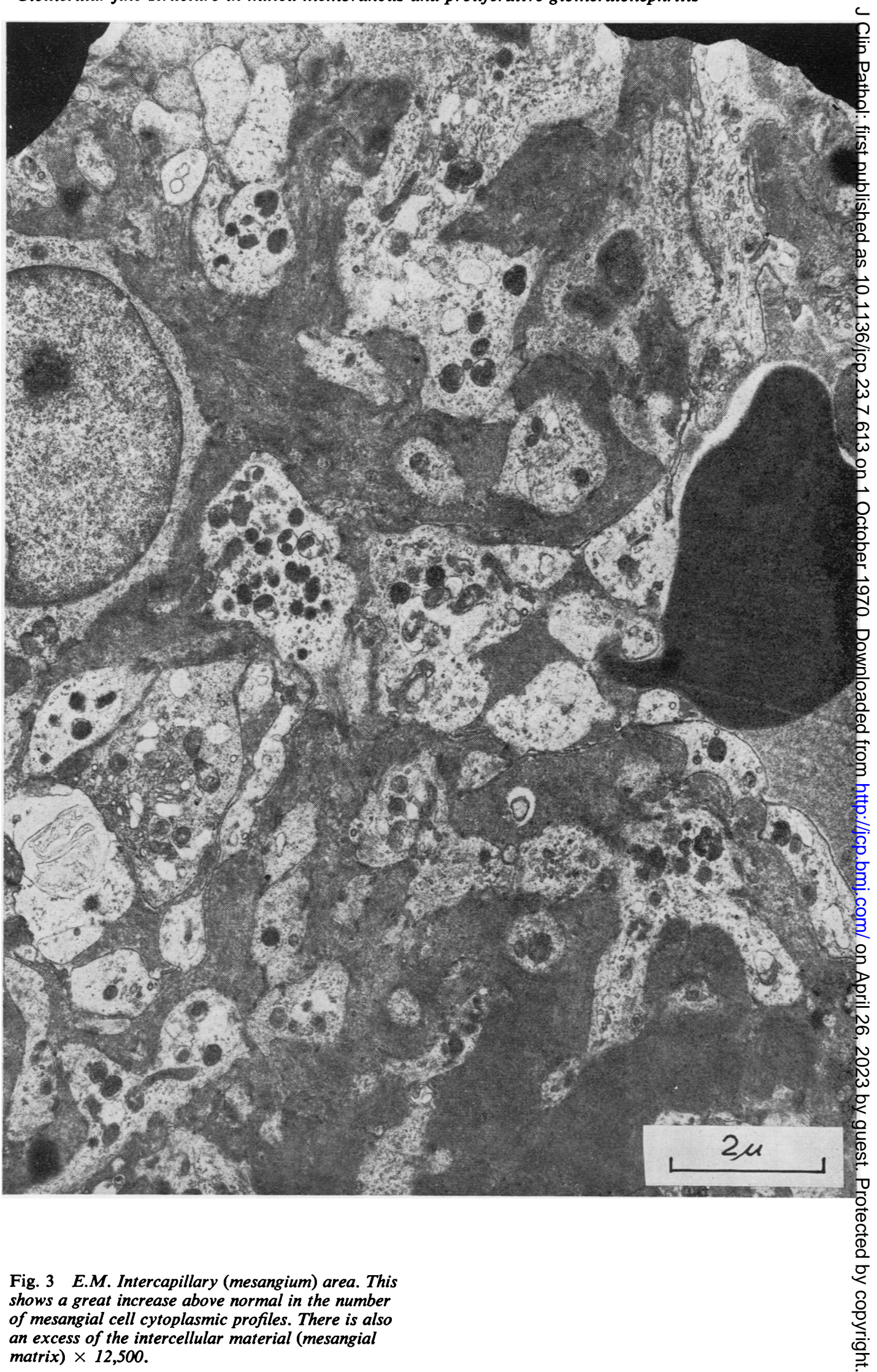




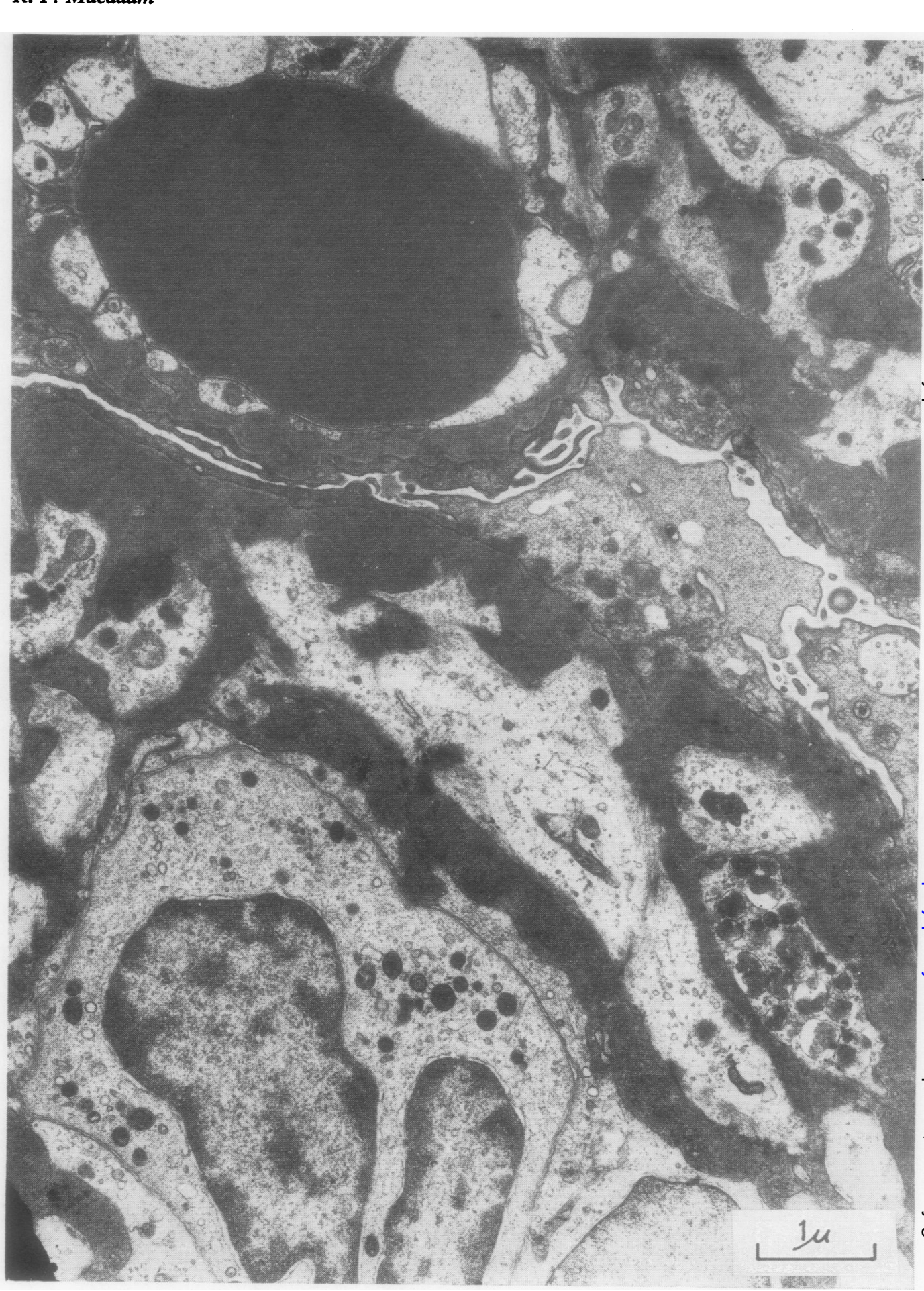

Fig. 4 E.M. Two adjacent glomerular capillaries. The increased density, variable width and irregular endothelial border of the basement membrane are illustrated. There is mesangial cell interposition in the basement membrane of the lower capillary 


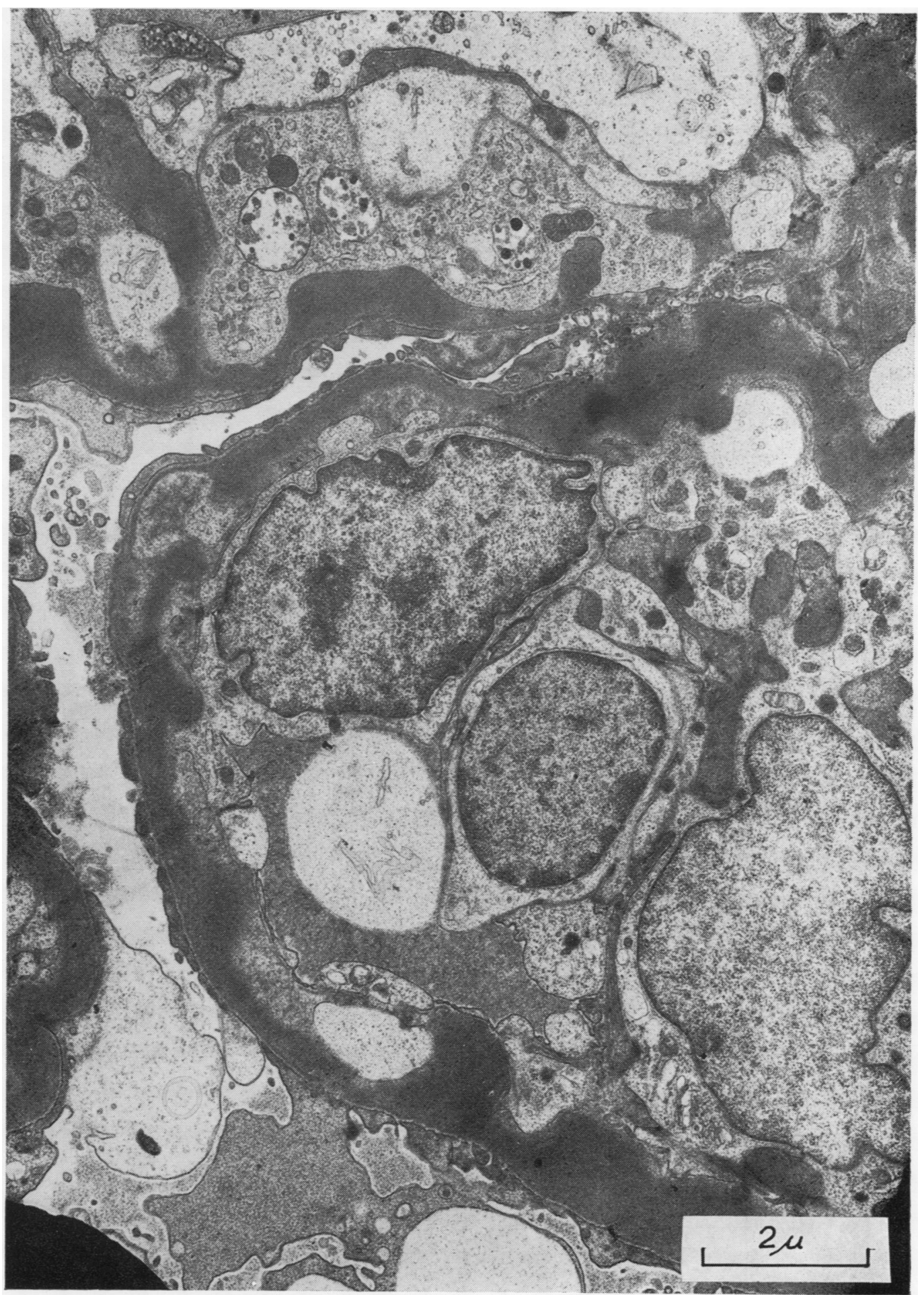

Fig. 5 E.M. Two adjacent glomerular capillaries. Both show the extreme variability in basement membrane thickness with areas of rarefaction. The upper capillary shows mesungial cell interposition and the lower one the capillary compression produced by mesangial cell hyperplasia $\times 12,500$. 


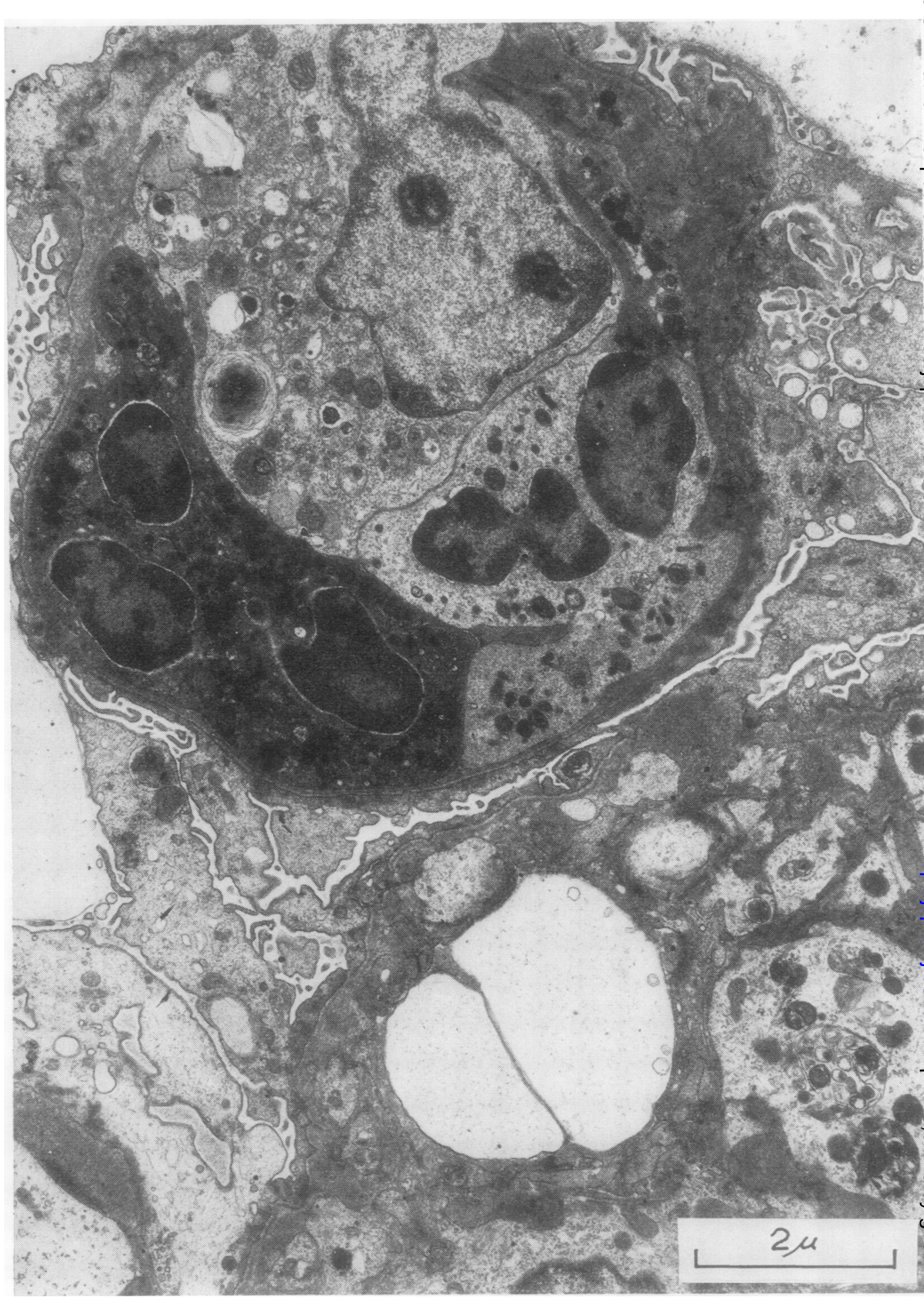

Fig. 6 E.M. A glomerular capillary occluded by two neutrophil polymorphonuclear leucocytes and a 


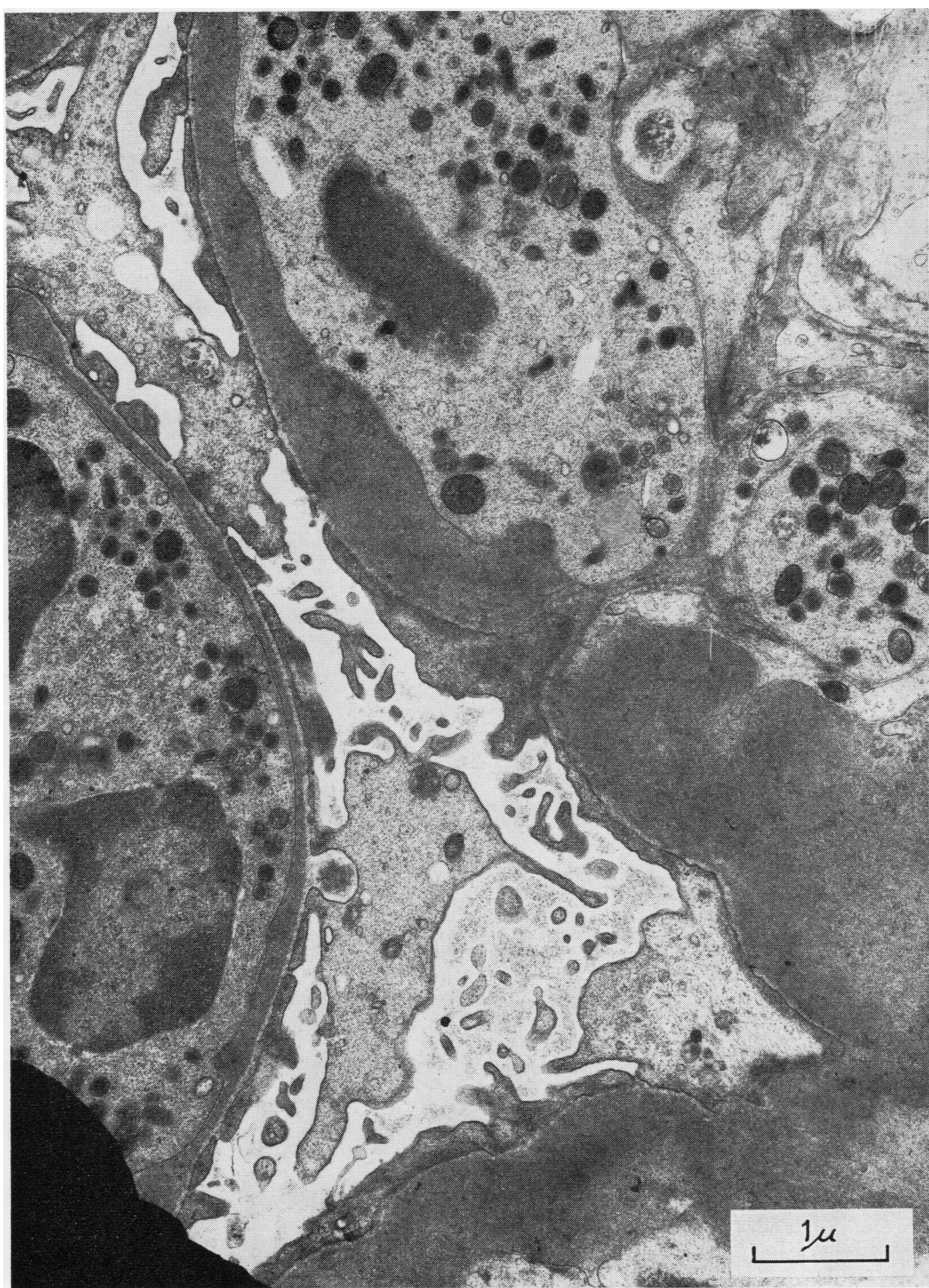

Fig. 7 E.M. Parts of two glomerular capillaries. The variably wide basement membrane is in direct contact with neutrophil polymorphonuclear leucocytes $\times 20,000$. 
(1961) described seven such cases under the heading of 'complex' or 'unclassified' glomerulonephritis and considered that this entity was probably a stage in the evolution of 'other forms' of glomerulonephritis. They, however, concluded that it was still a debatable point. Earle and Jennings (1961) considered that this histopathology could be a variant of a primarily proliferative lesion but added that it should be grouped separately until more information was available. There is no doubt, from the 1969 publications of Burkholder and Bradford and also of Arakawa and Kimmelstiel (1969), that this lesion does have a very obvious cellular proliferative element. What has not yet been shown is whether or not this type of picture includes the fine-structural complex known to the electron microscopist as indicative of complement-induced injury to the filtration basement membrane. The structura? result of interaction of serum complement with glomerular-bound antibody-antigen complexes is readily recognizable by electron microscopy iro classical acute poststreptococcal proliferative glomerulonephritis (Burkholder and Bradfords? 1969) and consists of the presence of multiple foci of endothelial cell stripped filtration basement membrane with direct contact betweers the latter structure and neutrophil polymor $\Phi$ phonuclear leucocytes. The mechanism postue lated for this result is the chemotactic effect? of interacting serum complement and glo? merular-bound antibody-antigen complex. Thus there is direct morphological evidence, in this case, of an immunologically mediated in flammatory process with mesangial cell hyperit plasia probably as a secondary phenomenon:

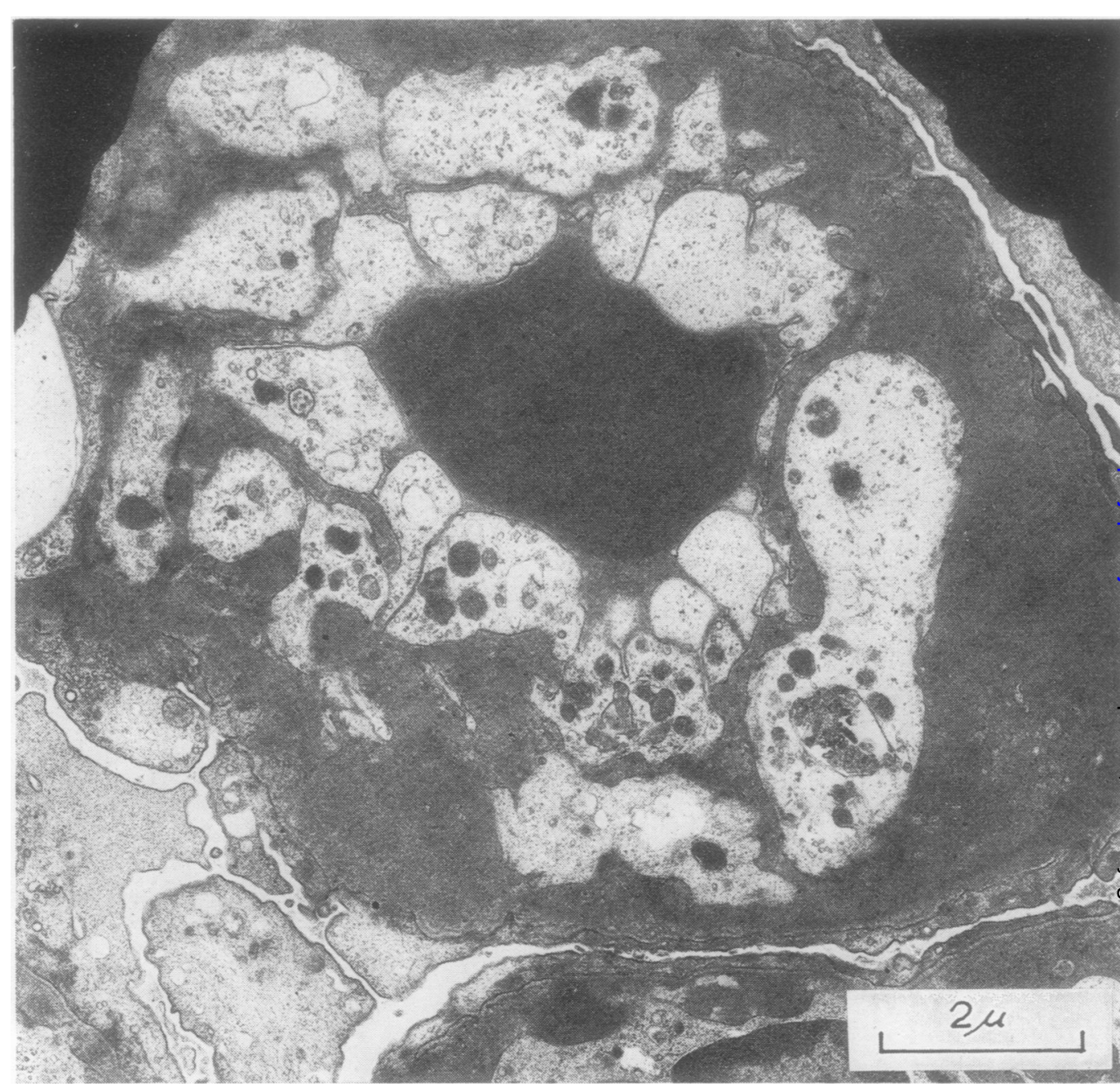

Fig. 8 E.M. Transverse section of glomerular capillary. This shows the great thickening of the basement membrane and the circumferential distribution of the mesangial interposition $\times 12,500$. 
Subepithelial deposits, characteristic of acute proliferative glomerulonephritis, are not present in this case. Perhaps this is accounted for by the 12-year duration of the disease. Such deposits were found in the five cases of Burkholder and Bradford (1969) but in none of them had the illness been present for more than eight years.

In addition to an increase in the number of mesangial cells in the glomeruli there is a striking penetration of the filtering basement membrane by mesangial cytoplasmic extensions. This phenomenon thus adds a third cellular layer to the capillary wall and contributes significantly to its thickness and non-homogeneous appearance by light microscopy. The enormous number of mesangial cell cytoplasmic units embedded within the mesangial matrix also accounts for the non- homogeneous appearance of the intercapillar zones. The intrusion of mesangial cytoplasmic processes into the filtering basement membrane is in the majority of capillaries, a complete circume ferential one. This remarkable invasive capacity of the mesangial cell has been noted, in varyin degree, in many states of disordered ren $\bar{q}$ function. Churg, Grisham, Goldstein, Yunis, an Porush (1965) described it in idiopathic nephroti迹. syndrome; Kimmelstiel, Osawa, and Berens (1967) described it in lobular glomerulonephritisp Faith and Trump (1966) described it in acute and chronic stage glomerulonephritis; Alousi, Pos? and Heymann (1969) described it in experimentat autoimmune nephritis in rats; and Arakawa and Kimmelstiel (1969) recorded the change in focad glomerulonephritis, light-negative lesions, Wegeniv

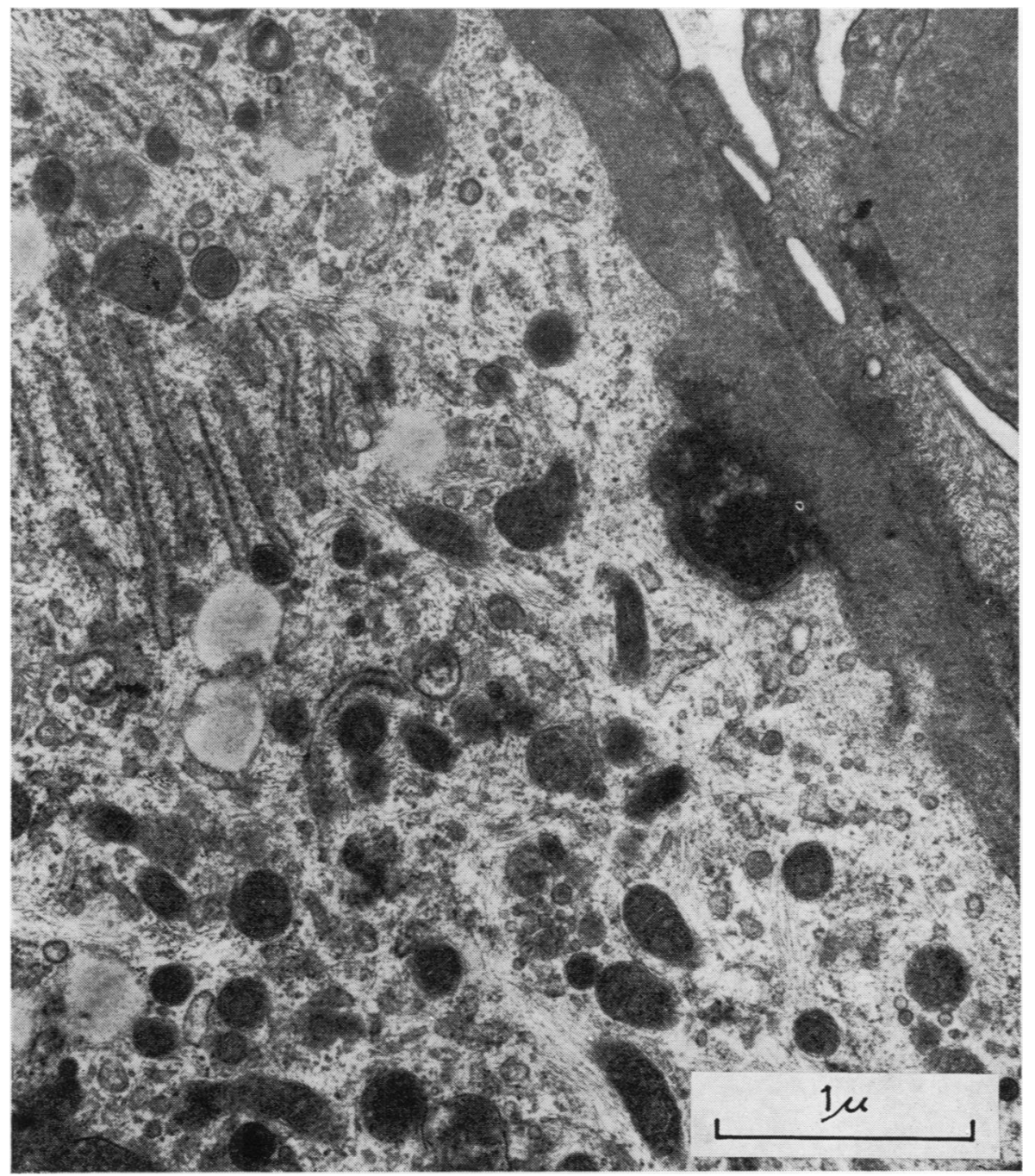

Fig. 9 E.M. Small part of glomerular capillary wall. A heterogeneous dense subendothelial deposit is illustrated $\times 30,000$. 
er's granuloma, toxaemia of pregnancy, diabetes mellitus, amyloidosis, and systemic lupus erythematosus. When the cell extensions do not encircle the capillary completely the change is called partial mesangial interposition, and when there is complete involvement, circumferential mesangial interposition (Arawaka and Kimmelstiel, 1969). These authors regard the finding of circumferential interposition as indicative of irreversible renal change but it is obviously not necessarily indicative of rapidly progressive disease. The mesangial cell participation in this lesion is of interest in two major respects. First, the mesangial interposition phenomenon in the filtering basement membrane is probably an expression of a basic glomerular capacity acquired early in phylogenetic development. Mesangial interposition is normal in the tadpole and frog (Galbraith, 1968) and the English sole (Bulger and Trump, unpublished observations). Mesangial cell hyperplasia and basement membrane hyperpermeability can be readily induced in the frog kidney (Galbraith, 1968) by the injection of uranyl nitrate. This rapidly causes glomerular capillary compression. Thus, mesangial cell stimulation and reaction may be early and important phenomena in proliferative glomerulonephritis and mesangial cell dysfunction may be related to altered permeability of the basement membrane.

It appears from this case and from the five childhood cases of Burkholder and Bradford (1969) that long survival is to be expected, despite the evidence of an active glomerular inflammatory process and severe morphological deformity of the nephron. This relatively benign course would argue against the use of immunosuppressive therapy in this now undoubtedly proliferative lesion.
I wish to thank Dr A. F. Lever for permission to $\frac{\varrho}{\overline{5}}$ study this biopsy.

References

Alousi, M. A., Post, R. S., and Heymann, W. (1969). Experi-O mental autoimmune nephritis in rats. Morphogenesis of the glomerular lesion: immunohistochemical and electron $\overline{\bar{s}}$ microscopic studies. Amer. J. Path., 54, 47-71.

Arakawa, M., and Kimmelstiel, P. (1969). Circumferential mesangial interposition. Lab. Invest., 21, 276-284.

Burkholder, P. M., and Bradford, W. D. (1969). Proliferative glomerulonephritis in children. A correlation of variedclinical and pathologic patterns utilizing light, immuno- $O$ fluorescence, and electron microscopy. Amer. J. Path., 56, 423-467.

Churg, J., Grishman, E., Goldstein, M. H., Yunis, S. L., and Porush, J. G. (1965). Idiopathic nephrotic syndrome in adults. A study and classification based on renal biopsies. New Engl. J. Med., 272, 165-174.

Earle, D. P., and Jennings, R. B. (1961). Post-streptococcal W glomerulonephritis. In Renal Biopsy, a Ciba Foundation. Symposium, edited by G. E. W. Wolstenholme and M. P.O Cameron, pp. 156-193. Little, Brown, Boston; Churchill, $\vec{\omega}$ London.

Faith, G. C., and Trump, B. F. (1966). The glomerular capillary을 wall in human kidney disease: acute glomerulonephritis, systemic lupus erythematosus and pre-eclampsia-eclampsia. Comparative electron microscopic observations and review. Lab. Invest., 15, 1682-1719.

Galbraith, S. L. (1968). Ultrastructure of the anuran kidney. B.Sc. Thesis in pure science. University of Glasgow.

Habib, R., Michielsen, P., de Montera, E., Hinglais, N., Galle. $\rightarrow$ P., and Hamburger, J. (1961). Clinical, microscopic and 6 electron microscopic data in the nephrotic syndrome of unknown origin. In Renal Biopsy, a Ciba Foundation Symposium, edited by G. E. W. Wolstenholme and M. P. Cameron, pp. 70-102. Little, Brown, Boston; Churchill. London.

Kark, R. M., Pirani, C. L., Pollak, V. E., Muehrcke, R. C., and Plainey, J. D. (1958). The nephrotic syndrome in adults: a common disorder with many causes. Ann. intern. Med. 용 49, 751-774.

Kimmelstiel, P., Osawa, G., and Beres, J. (1967). Some glomerular $\overrightarrow{\bar{\lambda}}$ changes by electron microscopy with predominant음 mesangial reaction. In Proceedings of the Third International 3 Congress of Nephrology, edited by R. H. Hepinstall, pp. 17-32. Karger, Basle.

Lawrence, J. R., Pollak, V. F., Pirani, C. L., and Kark, R. M. (1963). Histologic and clinical evidence of post-strepto coccal glomerulonephritis in patients with the nephrotic syndrome. Medicine (Baltimore), 42, 1-24. 